\title{
Bronchial Brushing Increases the Diagnostic Yield of Fiberoptic Bronchoscopy in Bronchogenic Carcinoma
}

Recep Bedir ${ }^{1}$, Servet Kayhan ${ }^{2 *}$, Ibrahim Şehitoğlu ${ }^{1}$, Halit Çınarka² ${ }^{2}$ Aziz Gümüş ${ }^{2}$, Aysel Kurt ${ }^{3}$, Hasan Güçer ${ }^{1}$, Cüneyt Yurdakul ${ }^{1}$, Ünal Şahin ${ }^{2}$

1.Departments of pathology, Recep Tayyip Erdogan University, School of Medicine, Rize, Turkey

2.Departments of chest disease, Recep Tayyip Erdogan University, School of Medicine, Rize, Turkey

3.Departments of thoracic surgery, Recep Tayyip Erdogan University, School of Medicine, Rize, Turkey

\begin{abstract}
Background: The importance of rapid and accurate cytopathological diagnosis in bronchial cancers is increasing due to advances in treatment modalities.

Aims: We evaluated the diagnostic methods and cytologic subtypes of bronchial cancers to determine the diagnostic reliability of different bronchoscopic techniques.

Material and methods: Retrospective data were obtained from the hospital files and pathological specimens of the patients with diagnosis of primary lung cancer from a period of 36 months. Cytological tumor typing was determined using histopathology of bronchoscopic forceps biopsy (FB), bronchial-bronchoalveolar lavage (BL), bronchial brushing (BB), transbronchial fine-needle biopsy. Computed tomography or ultrasonography guided transthoracic biopsy and surgical biopsies were used where the other interventional methods were inadequate for diagnosis.

Results: A total of 124 patients were diagnosed during study period. 119 (96\%) of them were male. The median age was 68 , ranging between 36 and 88 years. Histopathologic subtypes were determined as non-small cell carcinoma (NSCC) in 104 (83.9\%), squamous cell carcinoma in 64 (51.6\%), adenocarcinoma in 16 (12.9\%), NSCC not otherwise specified in 24 (19.3\%) and small cell carcinoma in $20(16.1 \%)$ patients. The combination of FB, BL and BB established the diagnosis of bronchogenic carcinoma in most of the cases (92.6\%).

Conclusions: Lung cancer is seen commonly in elderly male patients with smoking history and squamous cell carcinoma is the most common cytologic type. High diagnostic accuracy can be achieved by a combination of bronchoscopic FB, BB and BL procedures.
\end{abstract}

Keywords: Bronchoscopy; Lung cancer; Bronchial brushing

Recep Tayyip Erdogan University, School of Medicine, Department of Chest Disease, 53200 Rize, Turkey Phone +904642123009, Email: kayhanservet@gmail.com

Receive date: 2014-03-05 | Accept date: 2014-03-20 | Publish date:

2014-04-08 DOI: 10.7575/aiac.abcmed.14.02.02.05 


\section{Introduction}

Fiberoptic bronchoscopy is a safe and the most commonly used diagnostic method for obtaining a definite histopathological diagnosis of lung cancer. Forceps biopsy, bronchial brushing, bronchial-bronchoalveolar lavage and transbronchial fine-needle aspiration are used routinely as complementary diagnostic procedures, leading to accurate diagnosis in most of bronchogenic carcinomas (1).

In centrally located tumors, the diagnostic sensitivity of bronchial brushing was found

\section{Material and Methods}

Cytological materials of 124 patients with bronchogenic carcinoma treated in our clinic between January 2011 and December 2013 were used. Clinical and radiological data were collected from patients' files. Fiberoptic bronchoscopy was performed on 104 patients by skilled chest physicians in appropriate indications for lung cancer diagnosis. Informed consents of the patients were received before the procedures. Forceps biopsies were obtained from visible lesions. Bronchial brushes

\begin{tabular}{lc}
\hline Characteristics & No of cases (\%) \\
\hline Number of patients included in this study & $\mathbf{1 2 4}$ \\
\hline Gender & Male: $119(96 \%)$, female: $5(4 \%)$ \\
Age, median (range) & $68(36-88)$ year \\
\hline Localization of tumor & $\mathbf{n}(\%)$ \\
\hline Central lesions & $83(67 \%)$ \\
Peripheral lesions & $41(33 \%)$ \\
Endobronchial tumor stated & $103(83 \%)$ \\
\hline Histologic subtypes of lung cancer & $\mathbf{n}(\%)$ \\
\hline Non-small cell carcinoma & $104(83.9 \%)$ \\
Squamous cell carcinoma & $64(51.6 \%)$ \\
Adenocarcinoma & $16(12.9 \%)$ \\
Non-small cell carcinoma, not specified & $24(19.3 \%)$ \\
Small cell carcinoma & $20(16.1 \%)$ \\
\hline
\end{tabular}

Table 1: Basic patients and bronchoscopic data

between $44 \%$ and $94 \%$ and higher than that of bronchial lavage ranging between $27-90 \%$ in previous studies (2). However, the sensitivity of bronchial lavage and brushing decreases in peripherally located tumors, ranging between $6-83 \%$ and $4-43 \%$, respectively (2). These studies illustrate the need for improved interventional techniques for obtaining an early and accurate histopathological diagnosis of lung cancers.

The objective of the present study was to determine the diagnostic yield of single or combined bronchoscopic techniques for the diagnosis of lung cancer. were used for lesions not accessible to direct bronchoscopic forceps biopsies. We obtained at least three smears by bronchoscopic bronchial brush specimens using standard techniques and they were used for cytological examinations. Bronchial lavage was obtained by giving sterile saline into segmental or lobar bronchi of suspected areas for lung cancer. The specimens obtained by bronchoscopic biopsy, bronchial lavage and brushing were evaluated by experienced pathologists using HematoxylinEosin and Papanicolaou stains. 


\section{Results}

A total of 124, 119 men and 5 women were enrolled to this study (median age, 68; range, 36 to 88 years). Of these patients, 104 patients (83.8\%) underwent bronchoscopic biopsy, 7 patients $(5.6 \%)$ had transthoracic needle aspiration, and 13 patients (10.4\%) were sent for surgical resection for definite diagnosis. Basic patient and endoscopic data of the study groups are shown in Table 1.

Table 2: The sensitivity of diagnostic methods in lung cancer

\begin{tabular}{lcccccc}
\hline Result & \multicolumn{3}{c}{ Fiberoptic bronchoscopy } & \multirow{2}{*}{ Surgery } & \multirow{2}{*}{ TTB } \\
\cline { 2 - 5 } & $\mathrm{FB}$ & $\mathrm{BL}$ & $\mathrm{BB}$ & $\mathrm{TBB}$ & & \\
\hline $\mathrm{n}$ (positive/total) & $63 / 92$ & $43 / 75$ & $27 / 41$ & $21 / 32$ & $13 / 13$ & $7 / 7$ \\
\hline Sensitivity & $68.4 \%$ & $57.3 \%$ & $65.8 \%$ & $65.6 \%$ & $100 \%$ & $100 \%$ \\
\hline
\end{tabular}

FB: forceps biopsy, BL: bronchial lavage, BB: bronchial brushing, TBB: transbronchial biopsy, TTB: transthoracal biopsy

The basic diagnostic method for lung cancer was fiberoptic bronchoscopy and the sensitivity of diagnostic methods which were used in this study in lung cancer are shown in Table 2.

Definite cytologic typing and diagnosing of lung cancer was established by using three bronchoscopic techniques including forceps biopsy, bronchial brushing and bronchialbronchoalveolar lavage. The sensitivity of bronchoscopic techniques is shown in Table 3.

Table 3: The sensitivity of bronchoscopic methods in diagnosis of lung cancer

\begin{tabular}{lcc}
\hline $\begin{array}{l}\text { Bronchoscopic } \\
\text { procedure }\end{array}$ & $\begin{array}{c}\mathbf{n} \\
\text { (positive/total) }\end{array}$ & Sensitivity \\
\hline FB & $63 / 92$ & $68.4 \%$ \\
FB+BL & $67 / 75$ & $89.3 \%$ \\
FB+BL+BB & $38 / 41$ & $92.6 \%$ \\
\hline
\end{tabular}

FB: forceps biopsy, BL: bronchial lavage, BB: bronchial brushing

In addition to forceps biopsies, obtaining bronchial-bronchoalveolar lavage (Figure 1 and 2) and bronchial brushing (Figure 3,4,5 and 6) provided a higher diagnostic value to fiberoptic bronchoscopy.

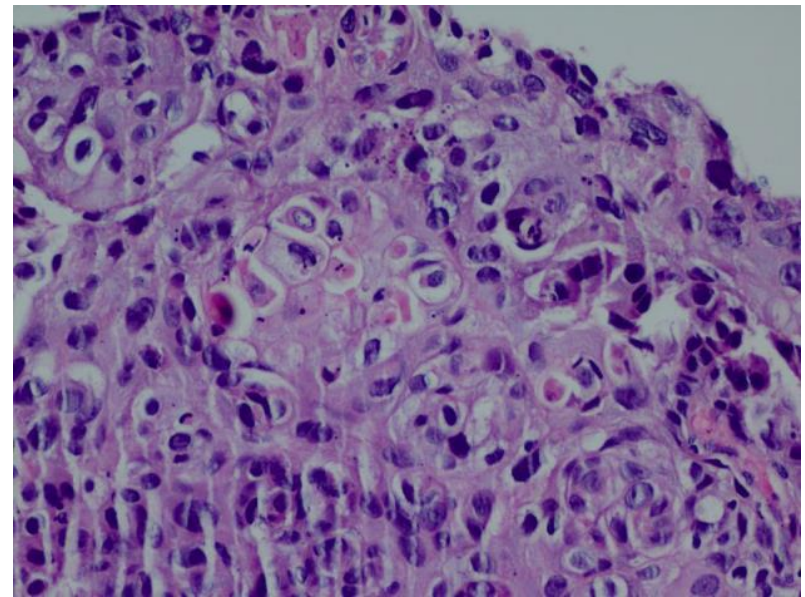

Figure 1: Cellular block of bronchial lavage showing atypical keratinized squamous cells (H\&Ex200). 


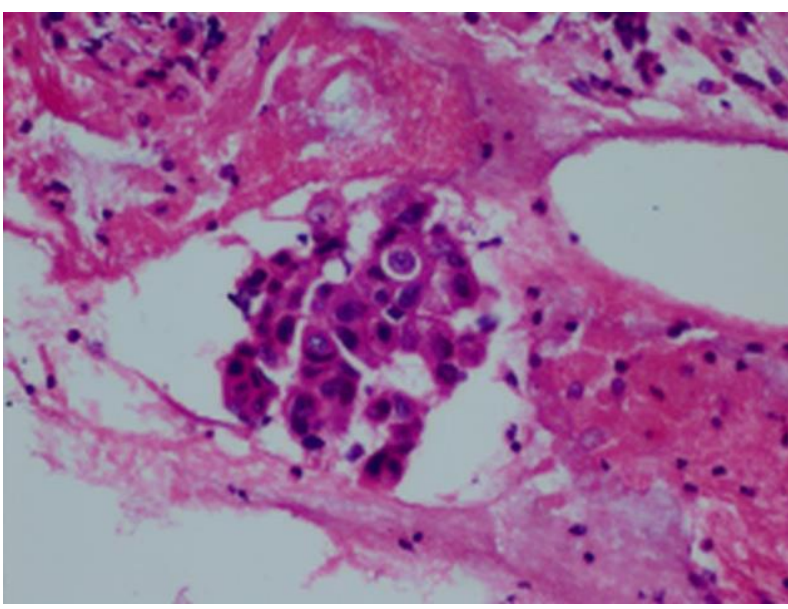

Figure 2: Cytological examination of bronchial lavage showing clusters of atypical squamous cells (H\&EX400).

\section{Discussion}

Flexible bronchoscopy is the first choice modality in the workup to establish a cytologic diagnosis in patients with suspected bronchogenic carcinoma. The previous studies supported the available bronchoscopic techniques for an accurate diagnosis of lung

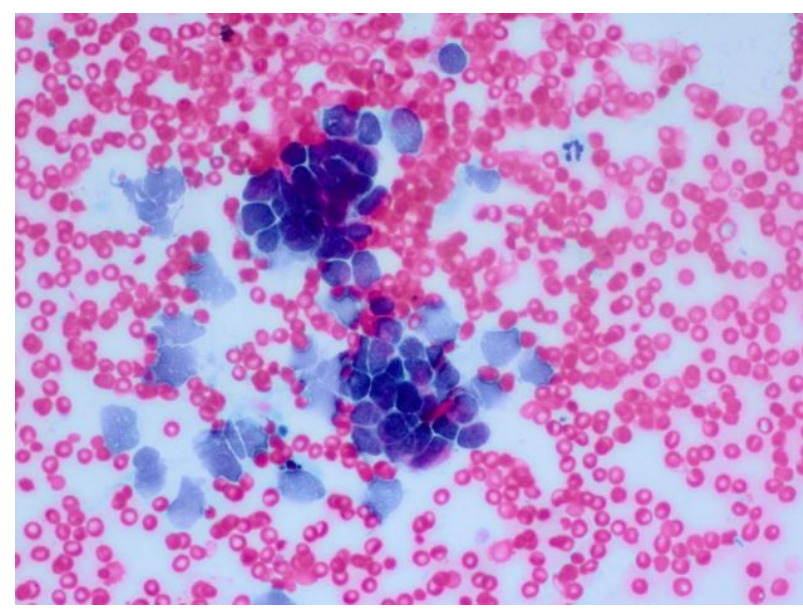

Figure 3: Bronchial brushing revealing a group of tumor cells showing uniform nuclear hyperchromasia, pleomorphism, irregularity of nuclear outline and nuclear molding showing small cell carcinoma (PAPx400).

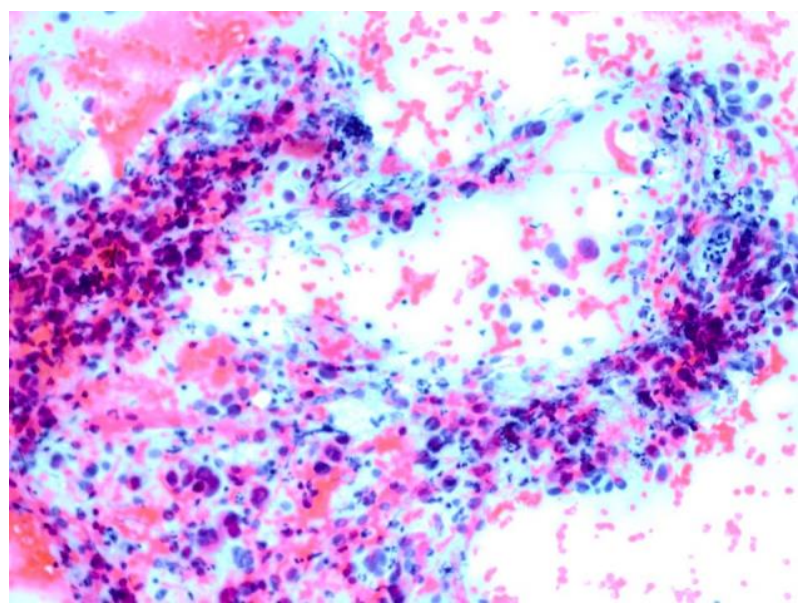

Figure 4: Bronchial brushing showing atypical keratinized squamous cells with hyperchromatic nucleus and marked nucleolus (PAPX200).

cancer (3). Most of the studies used the term of sensitivity or diagnostic yield regarding the flexible bronchoscopy in diagnosing the lung cancer $(4,5)$. The previous studies reported that the sensitivity of fiberoptic bronchoscopy was high for centrally located tumors and low for

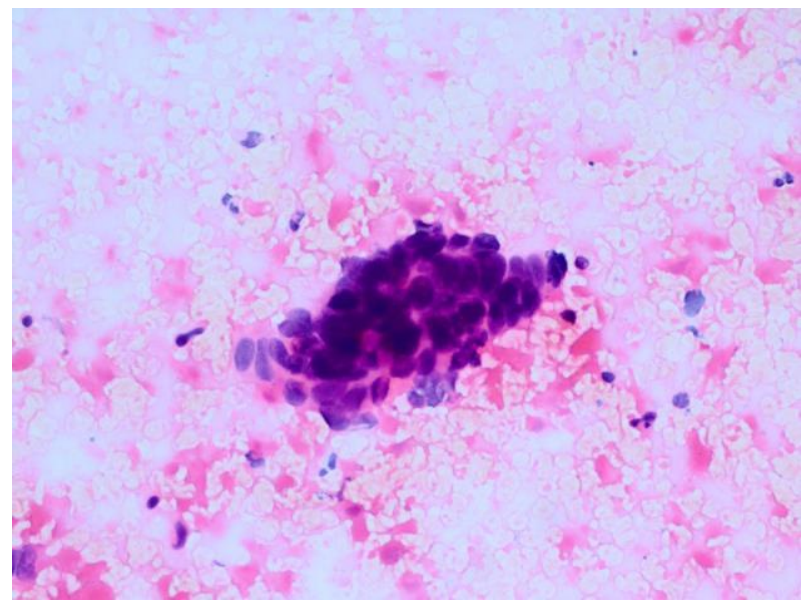

Figure 5: Bronchial brushing showing cohesive aggregates of atypical squamous cell showing nuclear hyperchromasia, nuclear enlargement and pleomorphism (H\&EX400). 


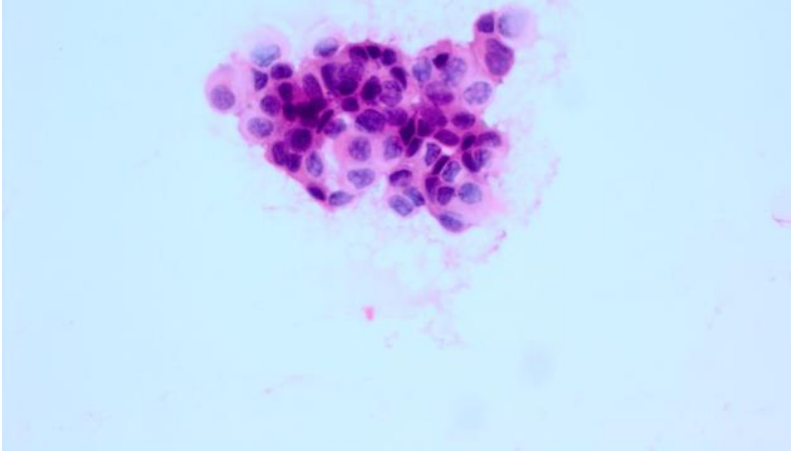

Figure 6: Bronchial brushing of adenocarcinoma showing sheets, acinar groups and a cluster of bronchial epithelial cells (H\&EX400).

peripherally located tumors (6). The results of our study demonstrated that obtaining multiple materials by different techniques is more sensitive than single or double methods for detecting lung cancer. A combination of bronchial brushing, bronchoalveolar lavage and forceps biopsy, especially in peripheral tumors, can improve the diagnostic sensitivity of fiberoptic bronchoscopy. These findings showed similar findings with the other studies (7).

A definitive diagnosis of bronchogenic carcinoma can be performed by cytologic examination, in concert with radiologic and clinical modalities. The overall sensitivity of combined bronchoscopic modalities was reported in the previous studies as 0.88 for centrally located tumors, where it was 0.69 for peripheral lesions. Nearly $20 \%$ of the cases with pulmonary lesions remains unresolved after bronchoscopic evaluations (3).

Endobronchial ultrasonography provides a better evaluation for peripheral pulmonary lesions and mediastinal lymphadenopathies. We did not use this diagnostic technique which could be considered the main limitation of this study.

The results of our study showed that obtaining several specimens by bronchial brushing, bronchoalveolar lavage and forceps biopsy combination is the best strategy in the definitive diagnosis of lung cancer via flexible bronchoscopy.

\section{Conflicts of interest}

The authors declare that they have no conflict of interest.

\section{References}

1. Jones AM, Hanson IM, Armstrong GR, O'Driscoll BR. Value and accuracy of cytology in addition to histology in the diagnosis of lung cancer at flexible bronchoscopy. Respir Med. 2001;95(5):374-8

2. Mazzone $\mathrm{P}$, Jain $\mathrm{P}$, Arroliga AC, Matthay RA. Bronchoscopy and needle biopsy techniques for diagnosis and staging of lung cancer. Clin Chest Med. 2002;23(1):137-58, ix.

3. Schreiber G, McCrory DC. Performance characteristics of different modalities for diagnosis of suspected lung cancer: summary of published evidence. Chest. 2003;123(1 suppl):115S-128S.

4. Sing A, Freudenberg N, Kortsik C, Wertzel H, Klosa B, Hasse J. Comparison of the sensitivity of sputum and brush cytology in the diagnosis of lung carcinomas. Acta Cytol. 1997;41(2):399-408.

5. Jay SJ, Wehr K, Nicholson DP, Smith AL. Diagnostic sensitivity and specificity of pulmonary cytology: comparison of techniques used in conjunction with flexible fiber optic bronchoscopy. Acta Cytol. 1980;24(4):304-12.

6. Baaklini WA, Reinoso MA, Gorin AB, Sharafkaneh A, Manian P. Diagnostic yield of fiberoptic bronchoscopy in evaluating solitary pulmonary nodules. Chest. 2000;117(4):1049-54.

7. Lam WK, So SY, Hsu C, Yu DY. Fibreoptic bronchoscopy in the diagnosis of bronchial cancer: comparison of washings, brushings and biopsies in central and peripheral tumours. Clin Oncol. 1983;9(1):35-42. 